\title{
Technical Issues and Challenges in Building Human Body Sensor Networks
}

\author{
Meghna Garg,Student \\ Computer Science \\ Chitkara University \\ Baddi(H.P), India
}

\author{
Manik Gupta,Professor \\ Computer Science \\ Chitkara University \\ Baddi(H.P), India
}

\begin{abstract}
In this research work, an exploration is done for identification of critical technical issues, problems, challenges in area of wireless body network sensors, which are continuous emerging as integral part of health monitoring systems. All this is possible due to the concept of 'Internet of Things' [5], in which day to day consumer devices, equipment are connected onto the network enabling information gathering and management of many vital signals. The first section gives introduction to wireless network developments in recent context, and then it is followed by discussion on existing work done in context of physical, Media access control and other aspects like energy consumption and security of such systems. The tabular summary on the gaps, limitations of Wireless body area network is done and based on this work; future directions are also suggested. Care has been taken to solicit high impact general paper for conducting this systematic study.
\end{abstract}

Keywords-Body Sensor Networks; MAC; Health Monitoring Systems; Internet of Things; Health Cloud; wireless biomedical sensor; wearable sensors; Energy optimization; motion-powered piezoelectric effect; Power consumption; time synchronization; Bandwidth utilization; memory; distributed storage; key management

\section{INTRODUCTION}

Physicians across the world are over loaded with vast amount of information from multi-sources, there are conflicting, incomplete diagnoses stories written and published. Most of this published material cannot really be reproduced and advancements in medical technology and patient health care remain a hit and trial and according to (WHO), World Health Organization, as many as one in ten patients in developed nation is harmed rather than cured in hospitals. All this can change for good, if continuous monitoring is done of patients using wireless network that help in not just clinical collaboration [10] but in responding in real time, on real time [6] patient's vital statistics. Progressive health care organizations are now adopting a policy of sharing, communicating in real time not just with other organizations but with the patient itself. The patient may be stationed at his/her home, office or is present in hospital itself. Now with the help of body sensor networks [1][2][4][5][19][23], heat stress [1], heart pulse [7][31], heartbeat [31], blood pressure [1][7][10] [15][20][22][31], blood sugar readings, sleep data[6][28][29] can be transmitted, and analyzed at multilocations for fast response. This wireless body sensor network, however are connected to 'Health Cloud [32]' for remote processing by computer algorithms and healthcare service providers. The wireless biomedical sensor forms a wireless body sensor network around human body enabling patient's vital signs to be collected and transmitted in a contextsensitive manner and same is applicable which observe health of animals[15], birds[15] etc. The data stream of these vital signs are stored in distributed storage systems like Hadoop[33] and are processed in Health cloud using algorithms that detects abnormality in them. However, like in all industries, there are challenges and issues which need to be taken care for it to be successful. The systems must be able to match health data compliance, privacy issues, secure connectivity with event synchronization and problems related to its rate of changes and size /volume of the data. There are many options that offer solutions to these problems and address the issues. These solutions include use of cloud based secure connectivity with backend storage solutions like Hadoop, this paper discusses further in this context in coming sections .

The paper discusses the issues and problems associated related to human body sensor network. The related work section discusses various aspects of Body sensor networks from basics to its construction and challenges faced by the industry. A tabular summary of problems is also given after that and last but not least discussion and future directions.

\section{RELATED WORK}

In this section, a systematic study is discussed in area of body sensor networks(BAN), the objective of this study is to arrive at, stage of learning where progress iterations done in this are covered for identification of the problems, issues, applications, algorithms involved in building body sensor networks. A network that can be build based on wearable sensors, implantable.

This research paper envisions collection of neurological high dimension; high resolution for brain imaging that is put for use in medical for mankind. The researchers are aiming here to build a toolkit that supports brain imaging data collection and analysis the brain process. The paper also throws light on other toolkits available in this area ; DETECT, EEGVIS, BCLLAB).It also discusses limitations of contempory efforts done in this context with respect to hardware. Limitations of high bandwidth requirement for building such long term, wearable systems of data collection from wireless networks attached to brain are discussed.

The paper points out, all though lot of research is happening in this area but many of these lack richness and 
depth of real world neuroimaging, hence their research is significant in this direction especially in direction of artifact like eyes movement (left blink, right blink), head rotation, jaw movements, etc. The paper also discusses the issue of developing minimal intrusive techniques/methods in detail.

[1] "Leveraging Knowledge From Physiological Data: OnBody Heat Stress Risk Prediction With Sensor Networks" This research work have focused on wearable sensors and have trained the empirical data generated by sensors with Bayesian net algorithm and decision tree and have achieved accuracy $92.1 \pm 2.91$ and $94.4 \pm$. The algorithm here, basically is predicting heat stress, $\mathrm{CO} 2$ level using body sensor network. Since, this algorithm is based on probability concepts the values of initial probability are provided and then based on the various conditions the value of probability is computed to arrive at the accuracy level. The graph (Figure 1) below shows skin temperature sample collection data using EOD suit.

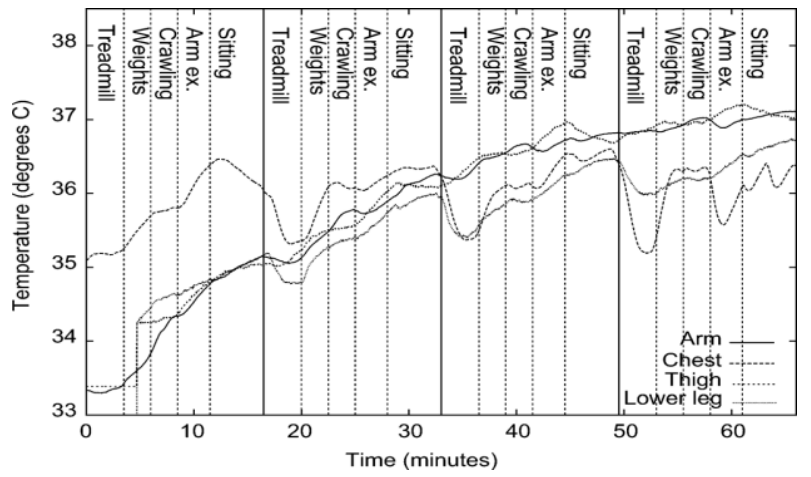

Fig. 1. Skin temperature data for a sample subject gathered during a mission like protocol while wearing an EOD suit. [1]

[4] "Energy-aware cross-layer optimization for EEG-based wireless monitoring applications" In this research paper energy optimization algorithm has been explained. The researchers have tried formulate an objective function that works to find an optimal tradeoff between the various design parameters along with constrains. The real idea is to minimize the total energy consumption subject to controls including data delay deadlines and distortion. The work is here about cross layer (Application-MAC-Physical). The result shows a delay deadlines increases energy consumption decreases. In graphs (Figure 2) below shows energy consumption patterns demonstrated in this research paper.

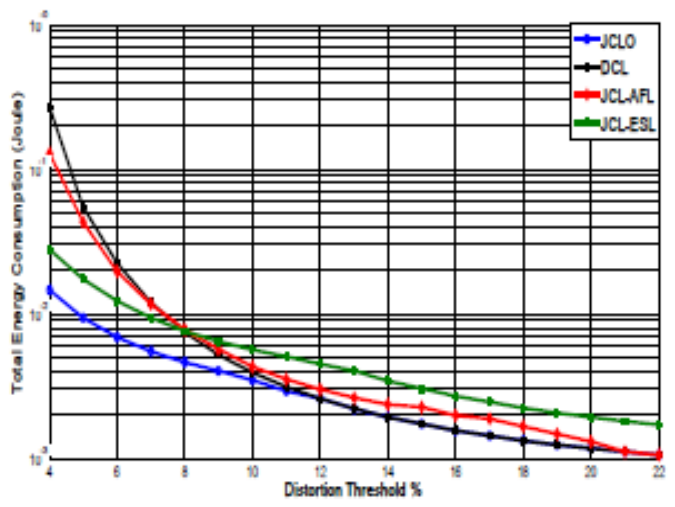

(a)

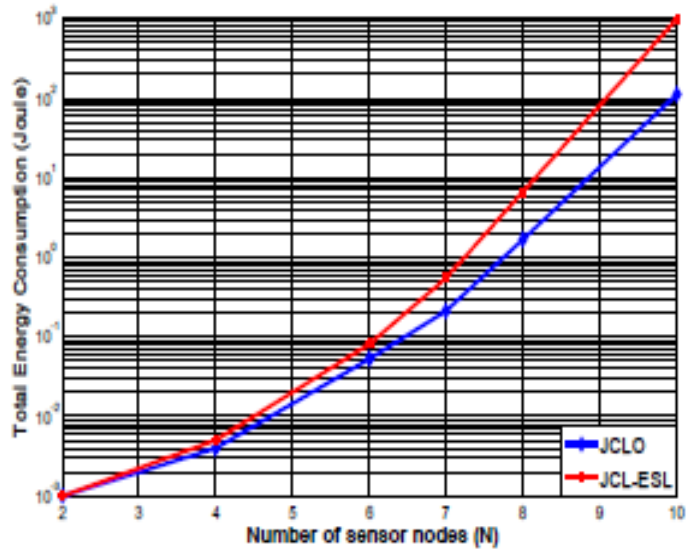

(b)

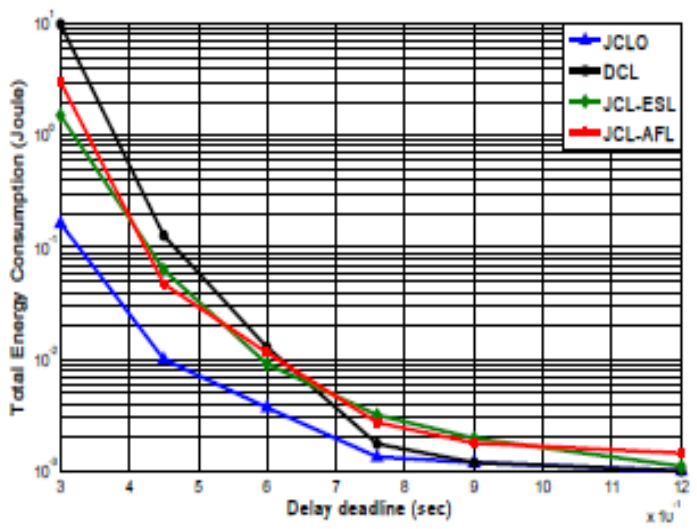

(c)

Fig. 2. A comparison between the total energy consumption using the proposed algorithms(a) For different distortion thresholds, (b) With increasing the number of sensor nodes and (c) For different delay deadlines[4]

[5] "A Motion-Powered Piezoelectric Pulse Generator for Wireless Sensing via FM Transmission" This paper demonstrates a process of building a motion-powered piezoelectric effect pulse generate for wireless sensing that using FM transmission can become a part of body sensor monitoring system. This device basically helps in energy harvesting attached with body of a subject. The paper shows engineering designs as well as mathematical relationship between the parameters that impact the performance of device like motion etc

[6] "Wireless Wearable Multisensory Suite and Real-Time Prediction of Obstructive Sleep Apnea Episodes" In this research work obstructive sleep apnea (OSA) dataset is used for classification using direct process based mixture Gaussian process (DPMG). The algorithm based can work for all body network sensors that can capture sleep data, from which a set of observation sleep apnea can be detected. The accuracy of this algorithm is $87 \%$. This paper demonstrates that how a supervised learning algorithm when accurately grouped can result in correctly predicted Obstructive Sleep Apnea episodes of a subject under observations. The bar graph (Figure 3) below shows the values of statistical variation of feature distribution of non and apnea cases. 


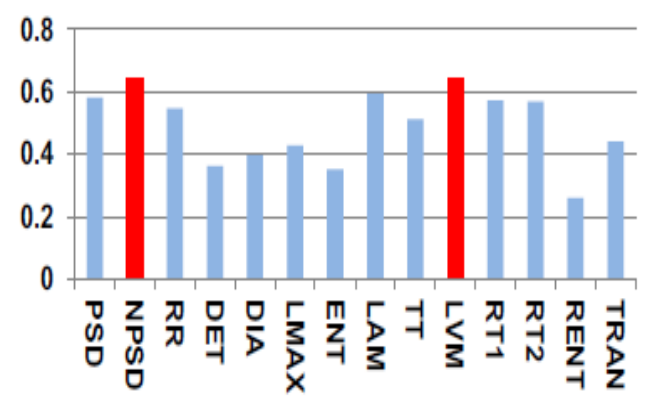

Fig. 3. KS Statistic variations of extracted feautures,KS ststistic indicates the maximal feature distribution differences between sleep apnea and nonapnea.[6]

[7] "A Neo-Reflective Wrist Pulse Oximeter" This paper illustrates wrist pulse oximeter building process with its performance evaluation. The design has 2 LED, or a receiver to capture wrist pulse signals from inner part of the wrist. It is improved version, true oximeter as compared to usual finger pulse oximeter. The calibration process shows second order relationship between ' $R$ ' value and oxygen saturation can provide better fit with the data, this is also illustrated in graph(Figure 4) below. The methods demonstrated here are based on linear and quadratic equation model of data fitting , and as per the graph values the curve made by quadratic equation model is doing the data fitting better as it is following non linear curve which is closer to the real life situation. This paper also helps us understand the internal working of body sensors in brevity.

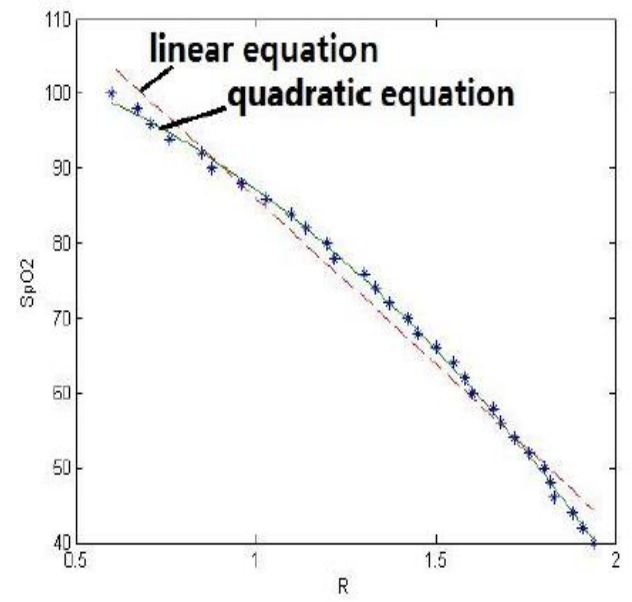

Fig. 4. The R curve modelled by two relationships[7]

[8] "An Attachable Clothing Sensor System for Measuring Knee Joint Angles" This research work shows that flexible natural based sensors can be incorporated in clothing, for measuring knee join angles. This research work was done on 10 subjects and the device showed only 1 of errors in measurements of angle. The (figure 5) shows the stress strain relationship captured using Knee Angle Sensor demonstrated in this research paper. From the graph it can be learned that initially the strain is changing faster into higher values when the strain is less $<0.5$. After this it moves higher in value but at slow rate and find it move up to marginally come down by few points.

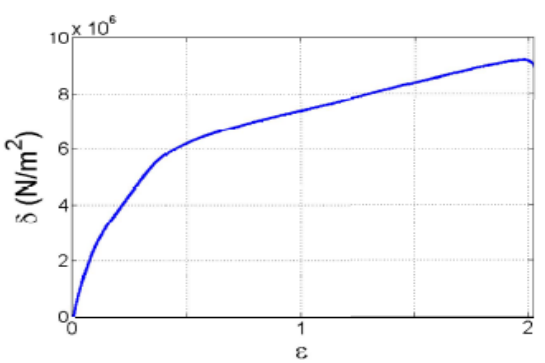

Fig. 5. Stress-strain curve obtained from the ACS sensor[8]

[9] "A Noncontact Capacitive Sensing System for Recognizing Locomotion Modes of Transtibial Amputees" This work shows usability of locomotion mode recognition system based on electromyography. The transtibial amputees with various levels of amputation have used for conducting evaluation experiments. Finally, the data is subjected to phase dependent quadratic and accuracy between $94-96 \%$ was obtained.

[10] "Predictive Monitoring of Mobile Patients by Combining Clinical Observations With Data From Wearable Sensors," This research work focused on demonstrating a combined system that monitors patient subjects using stimuli data collected from wearable sensors with clinical observations. The data is then processed with machine learning algorithms like probalistic training schemes like GMM etc.The results claimed in the paper show that these algorithms have high accuracy and true positive rates. However, the histograph below (figure 6) shows the frequency of two hundred patients with respect to their stay for the clinical study. Most of the patients stayed less than 10 days for the study and few more than 30 days.
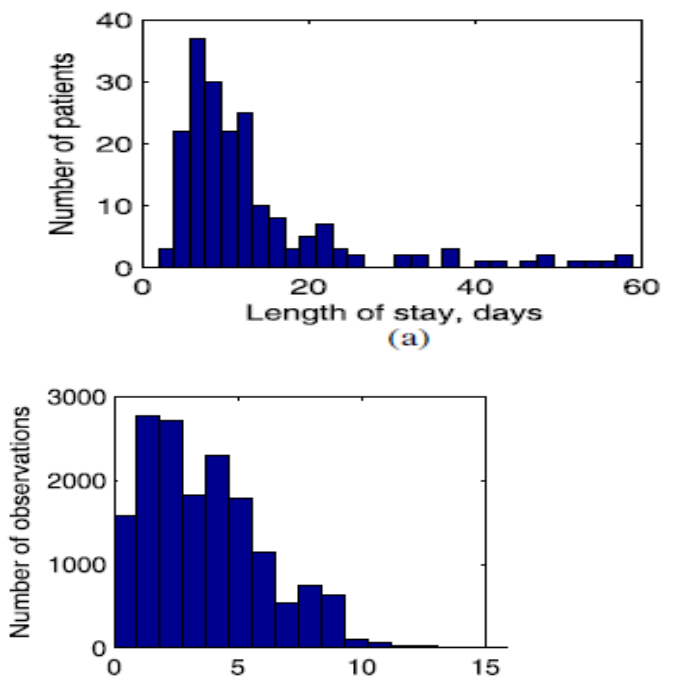

Time between observations, hours

(b)

Fig. 6. (a) Histogram of the length-of-stay of 200 studied patients in the Cancer Centre. (b) Histogram of time between manual observations, over all patients[10]

[12] "A Wearable Wideband Circularly Polarized Textile Antenna for Effective Power Transmission on a Wirelessly- 


\section{Powered}

Sensor

Platform,

This research paper shows simulation and experimental results of wideband wearable circulatory polarized textile antenna for low power transmission and it is battery less temperature sensor system that can be put in arm body. The results claimed in this paper show that the antenna performs really well with gain of 4.9Dblc.

[13] "Energy-Efficient Real-Time Human Mobility State Classification Using Smartphone," This research work tries to take advantage of the smart phone accelerometer sensors for human mobility analysis. The researcher are showing a framework based on a probabilistic algorithm that neutralizes the effect of different smart phones on body placements and orientation to allow human movements to be more accurately and energy efficiently identified. The method illustrated in this paper shows an accuracy of $92 \%$ when evaluated on a dataset, made of 15 subjects under study having a different urban mobility states like sit, stand, walk etc.

[15] "An Ingestible Sensor for Measuring Medication Adherence," This paper discusses a category of sensors that can be integrated with the oral dose of subjects and the sensors type is known as ingestible sensors. The sensors can be used for drug delivery, drug monitoring and to study drug efficiency. These sensors are micro/nano fabricated integrated circuits basically and the most important function they perform is to check medical adherence to other physiological metrics.

[16] "An adaptive home-use robotic rehabilitation system for the upper body," This paper introduces robotic prototype that mimics changes in subject is elbow angle in real-time, evaluation results show neural network based algorithm really performs well in this context of movement as error in movement, calibration etc is very low. In graph shown below the joint positions of the moving object are recorded. It can be seen that there is repetition of the movement positions, and as the graph (figure 7) is recorded the movement becomes smooth show rehabilitation.

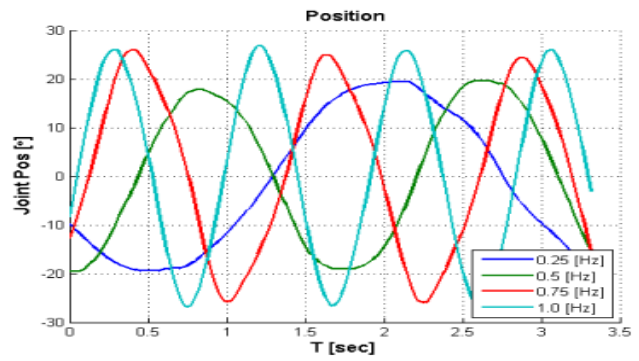

Fig. 7. Robot prototype joint angle by reference signal frequency[16]

[17] "EEG seizure detection and prediction algorithms: a survey" This paper discusses some state of art brain data seizure detection and prediction algorithms. The paper attempts to overcome the challenge of locating the seizure period in ECG recording which is done for analyzing epilepsy .The paper discusses 6 methods of seizure detection and predictions including time domain, wavelet domain, frequency domain, FCO and ICA domain. Empirical mode decomposition, summary the researcher have given a table which talks about methods, domain algorithms like time detection and prediction. Multi/single/channel database selfrecorded data, frame length, features used classifiers performance methods.

[18] "An advanced physiological data logger for medical imaging applications." The paper discusses the advances in physiological data logging technology (wired and wireless) for medical imaging applications, which involve use of microSD, USB, Bluetooth wireless technologies. The equipment demonstrated here can be used in wireless capsule endoscopy and skin temperature logging applications. The paper also discusses how images are captured which need to be processed to finally be logged for useful medical purposes.

[19][20] "A power efficient MAC protocol for wireless body area networks" These papers discusses MAC level protocol for wireless body sensor networks. The objective of this protocol for increase life time by reducing idle state power consumption and increase sleep time of a BAN node. The research claim this protocol is applicable for implanted, wearable sensor devices where data rate may high as in case of mp4 streaming or medium like in case of endoscope capsule.

[21] "Experimental characterizations of a UWB channel for body area networks "This paper discuses ultra wideband (UVB) technology that is used for wireless body area network. The results claimed in this research show that human body does not significantly degrade the impedance of monopole antenna. The measured path loss and multipath analysis suggest that this technology is excellent for low power, high rate transmission applications.

[22] "Modeling on-body dtn packet routing delay in the presence of postural disconnections" This paper discusses a stochastic framework for packet routing in body sensor networks. Delay modeling method is experimented, evaluated via simulation. The results show that on body sensor count can be safely be reduced without packet delivery delay due to store and packet routing mechanism depicted in this paper. This research work has also demonstrated the performance of the body sensor network in terms of packet delivery ratio, delay etc., which is graphically shown in the graphs below. The graphs (figure $8 \&$ figure 9 ) show comparative few of six algorithms.

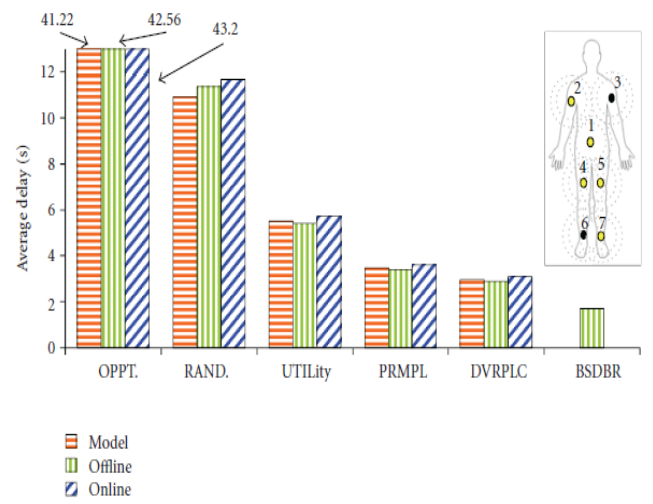

Fig. 8. On-body delivery delay for different DTN routing protocols[22] 


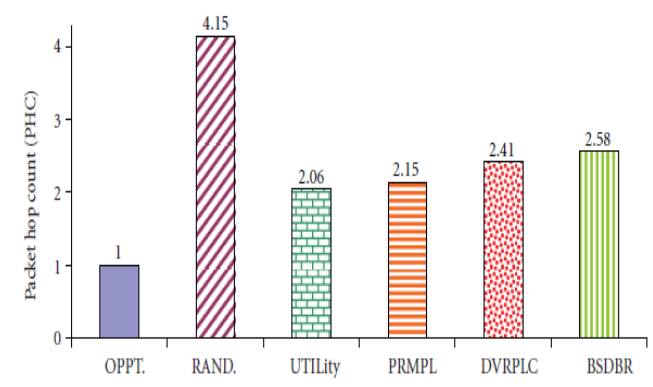

Fig. 9. Average packet hop count[22]

[23] "Design and analysis of an energy-saving distributed mac mechanism for wireless body sensor networks." This paper discusses the performance of distributed MAC process (DMAC) for wireless body sensor networks; this is done by incorporation of energy aware radio activation policies into the high performance of distributed queuing medium. The paper shows that energy assumption per bit of information vs relative traffic load increase slowly initially and later in after 0.7 value of relative traffic load increases exponentially as in (figure 10)

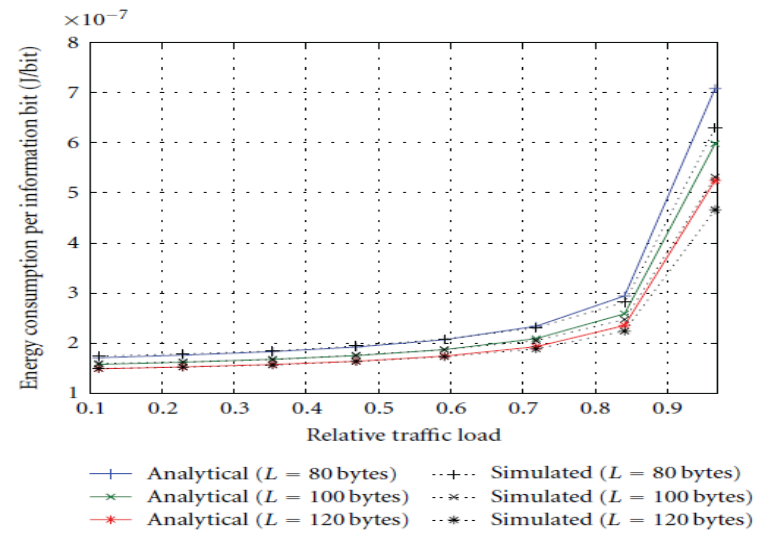

Fig. 10. DQ-MAC energy consumption per information bit:Analytical versus Simulation[23]

[26] "On PHY and MAC performance in body sensor networks." In this research work an empirical study has been conducted to evaluate the performance of body sensor implement communicating using radio. This paper tries to address the first concern which a person may have, which is observation is one thing, with body sensor device implanted in human body may lead to some side effects on the well being of a person, especially effect in body tissues. The researchers found that best performance of the sensor comes at $3 \mathrm{~cm}$ depth inside the liquid and not close to skin surface. Later section of this paper also shows simulation study of many low powered MAC protocols and cooperative view on them. In the graphs shown below, we can infer the relationship between the ERP and depth of liquid and RSSI and depth. But these graphs help us understand effectiveness of the sensor with respect to the depth of skin where the sensor is applied which can be validated from the behavior of ECC average coverage as shown in (figure 11,12,13)

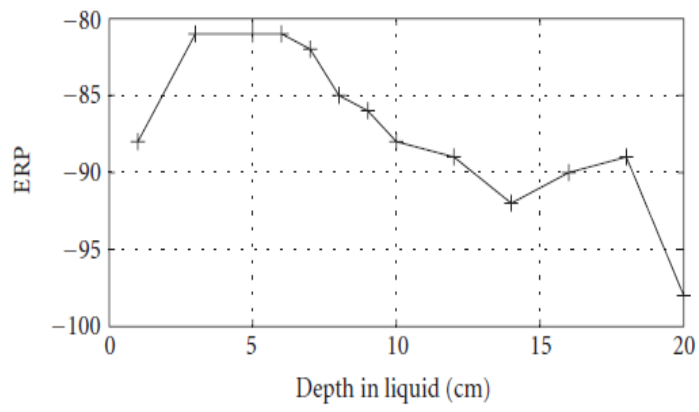

Fig. 11. ERP versus Depth in liquid[26]

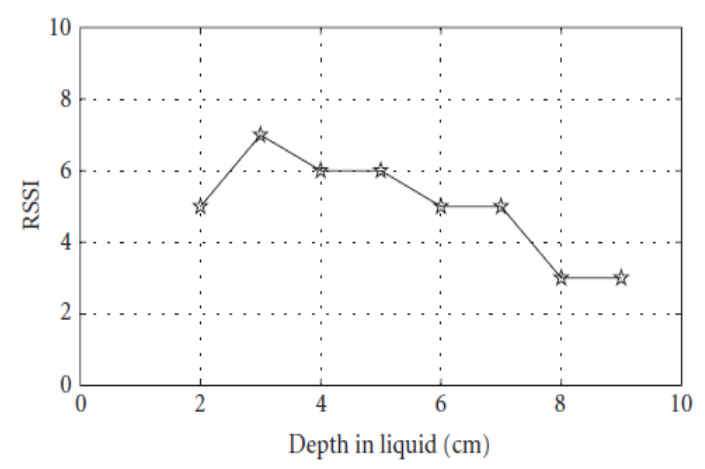

Fig. 12. RSSI versus Depth in liquid[26]

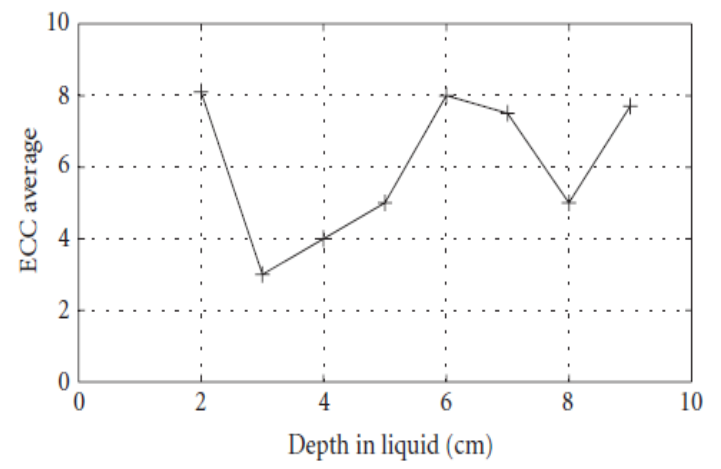

Fig. 13. ECC invocation versus Depth in liquid[26]

[30] "Biometric methods for secure communications in body sensor networks: resource-efficient key management and signal-level data scrambling." The paper highlights the challenges in developing resource efficient key management and signal level data scrambling for making it more secure. The researchers have used ECG signal for their study. The resulting simulation shows that efficacy increases with implementation of these key fusion methods.

\section{MAIN ISSUES AND CHALLENGES}

Based on the above systematic review of papers, materials associated with multiple aspects of emerging technologies in context of body sensors. We can identify following gaps, limitations of existing work done in this area as shown in Table No I. and illustrated in Figure 14. 
TABLE I. MAIN ISSUES AND CHALLENGES

\begin{tabular}{|c|c|c|}
\hline \multirow{2}{*}{ S.NO } & \multicolumn{2}{|r|}{ Main Issues And Challenges } \\
\hline & Parameter & Challenges \\
\hline 1. & Power Consumption[27] & The requirement is ultra low-powered devices. \\
\hline 2. & Sleep Cycle, Wakeup periods & $\begin{array}{l}\text { Synchronization of sleep and cycles with stimuli and with full body sensor } \\
\text { network. }\end{array}$ \\
\hline 3. & Idle listening time & This again is issue of synchronization of all devices as per stimuli. \\
\hline 4. & Overhead(Control packet) & $\begin{array}{l}\text { If the application of BAN is specific to particular type of stimuli. There is no } \\
\text { need for elaborate protocol . This way we can reduce protocol stack size there by } \\
\text { overheads. }\end{array}$ \\
\hline 5. & $\begin{array}{ll}\text { Packet } & \text { collision, } \\
\text { Retransmission[21][26] } & \end{array}$ & $\begin{array}{l}\text { If sudden outburst of stimuli. There is large volume of network traffic that must } \\
\text { flow smoothly so that packets reach without delay, without collisions, } \\
\text { retransmissions .It become challenge as routing soft components are frugal in } \\
\text { case of WBAN. }\end{array}$ \\
\hline 6. & Bandwidth utilization[21][26] & $\begin{array}{l}\text { Most of devices can handle small bytes, this limits the to and fro flow of data. } \\
\text { Hence, effective use Bandwidth is critical for such devices . }\end{array}$ \\
\hline 7. & Seizures of stimuli[17][2] & $\begin{array}{l}\text { Loading, recording the seizures of stimuli are very challenging in case of brain } \\
\text { and when body is in movement. }\end{array}$ \\
\hline 8. & $\begin{array}{l}\text { Storage of data collection, Time } \\
\text { lag[18][3][6] }\end{array}$ & The need is distributed storage. \\
\hline 9. & Memory & Limited to store and process. \\
\hline 10. & Signal Integrity & $\begin{array}{l}\text { Signal must maintain its coherence, shape, energy and spectrum properties and } \\
\text { no agent must interfere /manipulate it . }\end{array}$ \\
\hline 11. & Signal scrambling & $\begin{array}{l}\text { This can really secure signal, but at the same time this may lead to overload in } \\
\text { terms of delay and reduced synchronization. }\end{array}$ \\
\hline 12. & Signal shape & $\begin{array}{l}\text { No interference, no noise or distortion should be there when signal reaches } \\
\text { control room. }\end{array}$ \\
\hline 13. & $\begin{array}{l}\text { Key management, Key generation } \\
\text {, Key Exchange, Key recordability } \\
\text {,Key [27][30][22] }\end{array}$ & $\begin{array}{l}\text { Operational resources in body sensor networks are highly restricted, } \\
\text { incorporation of key management scheme lead to overhead, synchronization } \\
\text { issues at the lost of security which is also essential. }\end{array}$ \\
\hline 14. & Error in measurement of sensor[8] & $\begin{array}{l}\text { This depends upon the accuracy of digital instrument tool with which } \\
\text { measurements are taken both in terms of hardware and software components. }\end{array}$ \\
\hline 15. & $\begin{array}{l}\text { Area, Volume and Weight } \\
{[12][19][20][23]}\end{array}$ & $\begin{array}{l}\text { Unless nano, micro size is realized, there is limited scope of development in this } \\
\text { area, as a human body must not feel burden of sensors in terms of weight, area } \\
\text { ratio and volume. }\end{array}$ \\
\hline 16. & $\begin{array}{l}\text { Harmful effects of body sensors on } \\
\text { human tissues and overall well } \\
\text { being }\end{array}$ & $\begin{array}{l}\text { This technology's side effects still need to be observed and recorded for } \\
\text { understanding its ill effects on human body. }\end{array}$ \\
\hline 17. & Inert and Green technology[24] & $\begin{array}{l}\text { Body sensors must not react with human tissues and affect the health due to } \\
\text { radiation transmission of signal waves. }\end{array}$ \\
\hline 18. & $\begin{array}{l}\text { Integration of sensor data with } \\
\text { other clinical data.[10] }\end{array}$ & $\begin{array}{l}\text { This is critical for proper working and spirit of the concept of continuous } \\
\text { monitoring so that physician can take diagnostic decisions. }\end{array}$ \\
\hline 19. & $\begin{array}{l}\text { Integration to main stream medical } \\
\text { technology[10] }\end{array}$ & $\begin{array}{l}\text { Body sensors network data must be interoperable for it to be used across } \\
\text { departments and must be able to follow standards like HL7 etc. }\end{array}$ \\
\hline 20. & Time Synchronization[14][22] & $\begin{array}{l}\text { Final transmission to control room may lead to problems, challenges in terms of } \\
\text { delay, time lag and signal may require pre and post process. }\end{array}$ \\
\hline 21. & Detection & $\begin{array}{l}\text { In built anomaly detection, adversity identification systems are must, as these } \\
\text { may prone to DDOS, Sybil etc, attacks. }\end{array}$ \\
\hline 22. & Prediction & $\begin{array}{l}\text { Based on historical data, WBAN must have algorithm to predict health issues } \\
\text { like heart attack [31] for its real success and application. }\end{array}$ \\
\hline
\end{tabular}




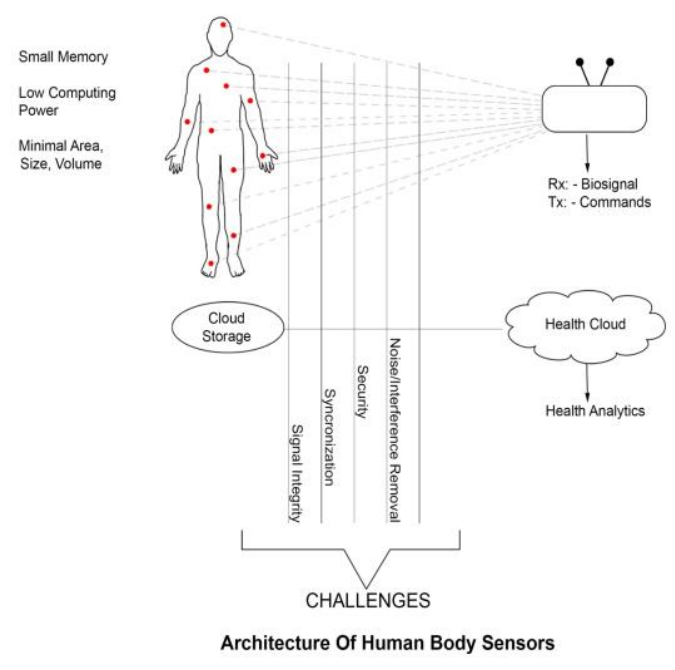

Fig. 14. Architecture of Human Body Sensors

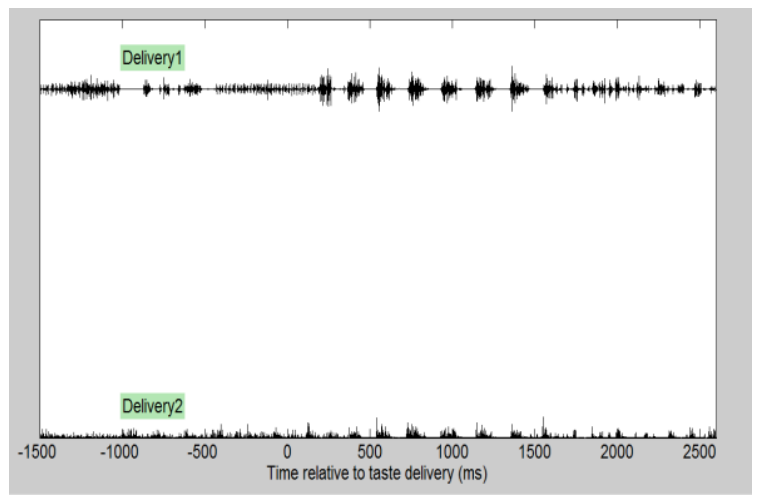

Fig. 15. Example of Human Body Sensors picking up Drug \& Taste stimuli[34]

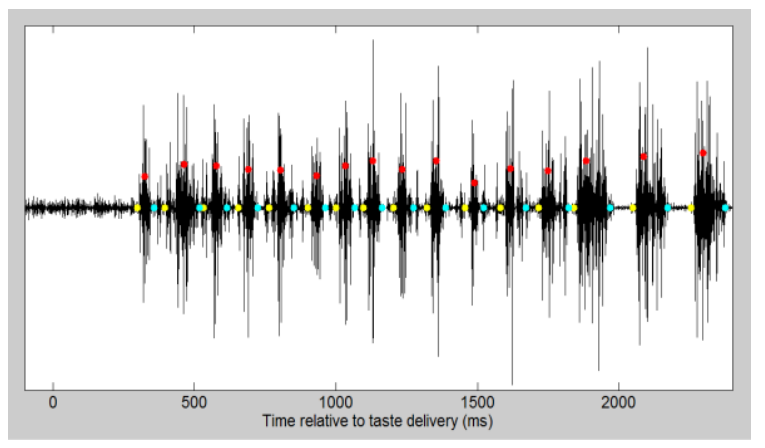

Fig. 16. Example of Human Body Sensors picking up Drug \& Taste stimuli[34]

The Figure 15 \& Figure 16 shows the EMG spike response when a drug shows its effect on the human subject and at the same time it shows the taste based body reaction /stimuli when the drug is tasted from brain captured by human body sensors.

\section{CONCLUSION AND DISCUSSION}

The applications are enormous of body sensor networks. They can be used right from the birth of a person till his/her old age.
Body sensor networks are now been developed for disease management and previous oriented healthcare. These systems may be synchronous or asynchronous in nature with multibody platform designs and implementation may help detect motor patterns, heat stress for example. Now, these networks are also now being experimented in understanding group dynamism like understanding vital signs of a cricket team. However, it should also be noted that, some of the implication of the problems are quite grave, if link interruptions or failures occurs in "internet of medical equipment" it can lead to unwanted consequences and other medical system complications.

Then, there are striking implications in case of data ownership in case we are using some fitness trackers and medical devices. Who should own the data related to the heartbeats? Person, community or some fitness device company.Even if the answer would seem obvious, but in today's environment it's not so clear. Existing technology in body network are not even addressing existing problems technically, and further development is likely to exacerbate that state of affairs due to inherent nature of Body sensor network technology.

In this research work, we have done systematic study for identification of applications, implications of the problems development in area of body sensor networks. The paper attempts to cover all that would impact the building, deployment and working of the body sensors in technical sense. The paper also covers MAC, physical layer issues as well as other hardware components, building locks used in build antennas for body sensors.

However, we have not covered discussion on the ethical issues related to Human Body sensors although, future similar work may cover up moral and ethical issues also.

\section{FutURE Direction IN BODY AREA NETWORK}

Future of WBAN lies in volume of implementations, installation and adoption of this technology by people at large. There is huge development, experimentation going on this context. In no time, these networks will become important tool in today's health. However, the challenges are also there, especially related to secure transmission and integrity of signals. For future directions, we suggest, artificial algorithms must be used for securing signal shapes and communication as well maintain integrity.

\section{ACKNOWLEDGMENT}

I would like to take this opportunity to thank my guide Manik Gupta, and my parents for helping me in the research study.

\section{REFERENCES}

[1] Gaura, E., Kemp, J., Brusey, J., "Leveraging Knowledge From Physiological Data: On-Body Heat Stress Risk Prediction With Sensor Networks," Biomedical Circuits and Systems, vol. 7, pp. 861-870, Dec. 2013.

[2] McDowell, K., Chin-Teng Lin, Oie, K.S., Tzyy-Ping Jung; Gordon, S.Whitaker, K.W., Shih-Yu Li, Shao-Wei Lu, Hairston, W.D., "RealWorld Neuroimaging Technologies," IEEE, vol. 1, pp. 131-149, 2013.

[3] Costlow, T., "Camera phone bans expected," Distributed Systems Online, vol. 5, pp. 5/1-5/3, Feb. 2004. 
[4] Awad, A., Hussein, R., Mohamed, A., El-Sherif, A.A., "Energy-aware cross-layer optimization for EEG-based wireless monitoring applications," Local Computer Networks (LCN), 2013 IEEE 38th Conference , pp. 356-363, Oct. 2013

[5] Hao Jiang, Kiziroglou, M.E., Yates, D.C., Yeatman, E.M., "A MotionPowered Piezoelectric Pulse Generator for Wireless Sensing via FM Transmission," Internet of Things Journal, IEEE, vol. 2, pp. 5-13, Feb. 2015

[6] Le, T.Q. ,Changqing Cheng, Sangasoongsong, A. , Wongdhamma, W.,Bukkapatnam, S.T.S., "Wireless Wearable Multisensory Suite and Real-Time Prediction of Obstructive Sleep Apnea Episodes," Translational Engineering in Health and Medicine, IEEE Journal, vol. 1, pp. 2700109-2700109, 2013

[7] Pang, G., Chao Ma, "A Neo-Reflective Wrist Pulse Oximeter," Access, IEEE , vol. 2, pp. 1562-1567, 2014

[8] Bergmann, J.H.M., Anastasova-Ivanova, S., Spulber, I., Gulati, V., Georgiou, P., McGregor, A., "An Attachable Clothing Sensor System for Measuring Knee Joint Angles," Sensors Journal, IEEE , vol. 13, pp.4090-4097, Oct. 2013

[9] Enhao Zheng, Long Wang; Kunlin Wei, Qining Wang, "A Noncontact Capacitive Sensing System for Recognizing Locomotion Modes of Transtibial Amputees," Biomedical Engineering, IEEE, vol. 61, pp. 2911-2920, Dec. 2014

[10] Clifton, L., Clifton, D.A., Pimentel, M.A.F., Watkinson, P.J.,Tarassenko, L., "Predictive Monitoring of Mobile Patients by Combining Clinical Observations With Data From Wearable Sensors," Biomedical and Health Informatics, IEEE Journal, vol. 18, pp. 722-730, May 2014

[11] Ngai, G., Stephen Cf Chan; Cheung, J.C.Y., Lau, W.W.Y., "Deploying a Wearable Computing Platform for Computing Education," Learning Technologies, IEEE, vol. 3, pp. 45-55, Jan.-March 2010

[12] Lui, K.W., Murphy, O.H., Toumazou, C., "A Wearable Wideband Circularly Polarized Textile Antenna for Effective Power Transmission on a Wirelessly-Powered Sensor Platform," Antennas and Propagation, IEEE , vol. 61, pp. 3873-3876, July 2013

[13] Oshin, T.O. ,Poslad, S., Zhang, Z., "Energy-Efficient Real-Time Human Mobility State Classification Using Smartphones," Computers, IEEE , vol. PP, pp. 1-1

[14] Goth, G., "Delay-Tolerant Network Technologies Coming Together," Distributed Systems Online, IEEE, vol. 7, pp. 2,2, Aug. 2006.

[15] Hafezi, H., Robertson, T.L., Moon, G.D., Kit-Yee Au-Yeung; Zdeblick, M.J., Savage, G.M., "An Ingestible Sensor for Measuring Medication Adherence," Biomedical Engineering, IEEE, vol. 62, pp. 99-109, Jan. 2015

[16] Dowling, A.V., Barzilay, O., Lombrozo, Y., Wolf, A., "An adaptive home-use robotic rehabilitation system for the upper body," Translational Engineering in Health and Medicine, IEEE Journal, vol. 2, pp. 1-10, 2014.

[17] Alotaiby, Turkey N., Saleh A. Alshebeili, Tariq Alshawi, Ishtiaq Ahmad, and Fathi E. Abd El-Samie. "EEG seizure detection and prediction algorithms: a survey." EURASIP Journal on Advances in Signal Processing ,2014.

[18] Khan, Tareq Hasan, and Khan A. Wahid. "An advanced physiological data logger for medical imaging applications." EURASIP Journal on Embedded Systems, pp. 1-1, 2014.

[19] Koulali, Mohammed-Amine, Abdellatif Kobbane, Mohammed El Koutbi, Hamidou Tembine, and Jalel Ben-Othman. "Dynamic power control for energy harvesting wireless multimedia sensor networks."
EURASIP Journal on Wireless Communications and Networking, pp. 1-8, 2012,

[20] Al Ameen, Moshaddique, Niamat Ullah, M. Sanaullah Chowdhury, SM Riazul Islam, and Kyungsup Kwak. "A power efficient MAC protocol for wireless body area networks." EURASIP Journal on Wireless Communications and Networking, pp. 1-17, 2012.

[21] Xia, Lingli, Stephen Redfield, and Patrick Chiang. "Experimental characterization of a UWB channel for body area networks." EURASIP Journal on Wireless Communications and Networking, 2011.

[22] Quwaider, Muhannad, Mahmoud Taghizadeh, and Subir Biswas. "Modeling on-body dtn packet routing delay in the presence of postural disconnections." EURASIP journal on wireless communications and networking, 2011.

[23] Begonya, Otal, Alonso Luis, and Verikoukis Christos. "Design and analysis of an energy-saving distributed mac mechanism for wireless body sensor networks." EURASIP Journal on Wireless Communications and Networking, 2010.

[24] Naveen, Chilamkurti, Zeadally Sherali, Jamalipour Abbas, and Das Sajal K. "Enabling Wireless Technologies for Green Pervasive Computing." EURASIP Journal on Wireless Communications and Networking 2009 , (2010).

[25] Kolar, Anthony, Olivier Romain, Jade Ayoub, David Faura, Sylvain Viateur, Bertrand Granado, and Tarik Graba. "A system for an accurate 3D reconstruction in video endoscopy capsule." EURASIP journal on embedded systems , 2009 .

[26] Sana, Ullah, Higgins Henry, Islam SM Riazul, Khan Pervez, and Kwak Kyung Sup. "On PHY and MAC performance in body sensor networks." EURASIP Journal on Wireless Communications and Networking, 2009.

[27] Kaur, Jasdeep, and Sandeep Singh Gill. "QoS based energy efficient key management in body sensor networks." In Medical Imaging, m-Health and Emerging Communication Systems (MedCom),IEEE , pp. 14-19 , 2014.

[28] Chen, Min, Sergio Gonzalez, Athanasios Vasilakos, Huasong Cao, and Victor C. Leung. "Body area networks: A survey." Mobile networks and applications , pp. 171-193, 2011.

[29] Latré, Benoît, Bart Braem, Ingrid Moerman, Chris Blondia, and Piet Demeester. "A survey on wireless body area networks." Wireless Networks , pp. 1-18, 2011.

[30] Bui, Francis Minhthang, Dimitrios Hatzinakos. "Biometric methods for secure communications in body sensor networks: Resource-efficient key management and signal-level data scrambling." EURASIP Journal on Advances in Signal Processing , 2008

[31] Huaming Li, Student Member, IEEE, and Jindong Tan, Member, IEEE" Heartbeat-Driven Medium-Access Controlfor Body Sensor Networks" IEEE transactions on information technology in biomedicine, vol. 14, Jan. 2010

[32] Misra, S., Das, S.; Khatua, M., Obaidat, M.S. "QoS-Guaranteed Bandwidth Shifting and Redistribution in Mobile Cloud Environment", Cloud Computing, IEEE , vol. 2 , pp. 181 - 193 , AprilJune 2014

[33] Sobhy, D. , Coll. of Comput. Eng., Arab Acad. of Sci. \& Technol. \& Maritime Transp., Alexandria, Egypt, El-Sonbaty, Y., Abou Elnasr, M. "MedCloud: Healthcare cloud computing system" IEEE , pp. 161 - 166, Dec. 2012.

[34] Li, Jennifer X., Takashi Yoshida, Kevin J. Monk, and Donald B. Katz. "Lateral hypothalamus contains two types of palatability-related taste responses with distinct dynamics." The Journal of Neuroscience 33, pp. 9462-9473, 2013. 